\title{
Toward the Next Level of PCB Usage in Power Electronic Converters
}

\author{
Erik C. W. de Jong, Member, IEEE, Braham J. A. Ferreira, Fellow, IEEE, and Pavol Bauer, Senior Member, IEEE
}

\begin{abstract}
A means for power electronics to exploit the level of 3-D packaging already being implemented in compact consumer products, such as digital cameras, is investigated in order to increase its power density. The increase in functionality and usage of printed circuit board (PCB) in power electronic converters is highlighted and improvements proposed to boost $\mathrm{PCB}$ usage to the next level. The material and manufacturing cost of an embedded planar transformer in a PCB-assembled power converter has been substantially reduced by introducing flexible-foil PCB to create the many windings without increasing the remaining number of (expensive) rigid PCB layers. Aspects such as the required folding pattern and its PCB material usage receive qualitative and quantitative attention. Furthermore, increasing of PCB functionality as regards integration of passives, geometrical packaging, and 3-D thermal management enhancement has been addressed. Not only is it shown that the integration of passives into a single, multifunctional PCB transformer structure is feasible but also that the same integral PCB can be used to geometrically package the remaining bulky, low-frequency, discrete components to create a power-dense converter and enhance the 3 -D thermal management. A power density improvement of $66 \%$ (from 150 to $250 \mathrm{~W} / \mathrm{L}$ ) is achieved by the technology demonstrator.
\end{abstract}

Index Terms-Design methodology, geometrical packaging, optimization methods, packaging, power conversion, power density, power supplies, printed circuits, printed circuit layout.

\section{INTRODUCTION}

$\mathbf{T}$ HE MINIATURIZATION of electronic consumer products bring about many advantages for the user in terms of portability (weight and size) or in terms of functionality (more functions in the same volume). This miniaturization, however, requires a revolutionary change in the conventional design and packaging philosophy of such products, one which reaches beyond the 2-D domain into the 3-D domain.

The microelectronic industry is well ahead in this regard as can be seen from the 3-D packaging concepts applied to the electronic circuitry found in modern digital cameras [see Fig. 1(a)] or with high-density IC packaging [see Fig. 1(b)], for example.

Power electronics has yet to exploit this level of 3-D packaging to increase power density in converters intended for flatpanel monitors, PC peripherals, or even energy-saving light bulbs, for example. Printed circuit board (PCB) assembled power converters, in particular, have a distinct advantage as

Manuscript received November 29, 2006; revised August 3, 2007 and April 10, 2008; accepted July 1, 2008. Current version published December 9, 2008. Recommended for publication by Associate Editor E. Santi.

E. C. W. de Jong was with Delft University of Technology, Delft 2628 CD, The Netherlands. He is now with KEMA Netherlands B.V., Arnhem 6800 ET, The Netherlands (e-mail: erik.dejong@kema.com).

J. A. Ferreira and P. Bauer are with Delft University of Technology, Delft 2628 CD, The Netherlands (e-mail: j.a.ferreira@tudelft.nl; p.bauer@tudelft.nl).

Digital Object Identifier 10.1109/TPEL.2008.2004276 they share the enabling technology used in the compact designs shown before: the $P C B$. The PCB itself has matured far beyond its initial purpose of electrical interconnection: it allows packaging in 3-D (Fig. 1) by using flexible foils [3], allows integration of electromagnetic functionality (passives integration) by implementing enhanced substrate materials [4], and also enhances thermal management by means of thermally conducting layers in intimate contact with the heat source [5], [6]. Exploiting this additional functionality of the PCB in power electronic converter design is the next challenge; therefore, this paper details the steps necessary to proceed to the next level of PCB usage in power electronics. Although the proposed technology is still in its infancy, this paper contributes in delivering a concise reference to assist the different mind-set required to design power electronics with the full advantage of its inherent PCB.

The first step in achieving this, discussed in Section II, addresses the increased functionality of PCB in the implementation of embedded planar transformers within a PCB-assembled converter. It investigates means to optimize PCB material usage, and thereby reduce the cost and complexity of its manufacturing process. The second step, discussed in Section III, increases the functionality of the PCB by integrating all the power passives of a resonant converter topology into the PCB (no additional materials or technologies required). It also introduces a design approach to geometrically package bulky, odd-shaped, discrete components in a compact 3-D shape using rigid-flex PCB. Furthermore, it also describes the 3-D thermal management enhancements using PCB technology to successfully combat the increase in loss density associated with the increase of power density. The resulting converter based on this multifunctional PCB technology is then quantitatively evaluated in Section IV.

\section{Optimal Usage of PCB Real Estate}

The demand for power density within power supplies and the steady increase in power-processing frequency have seen bulky, low-frequency, and discrete components make way for compact, high-frequency components, often bringing about unexpected benefits such as easier manufacturing and the possibility for functional integration. ${ }^{1}$ Planar transformers is one example hereof. Planar transformers are a popular choice in high-frequency power converters as it not only increases the converter's power density but also facilitates better thermal management due to its low-profile form-factor as well [7], [8]. They can be embedded into the converter's PCB by placing the

\footnotetext{
${ }^{1}$ More on the evolution from conventional component technology toward the advanced methods discussed herein can be found in [15] and [17].
} 


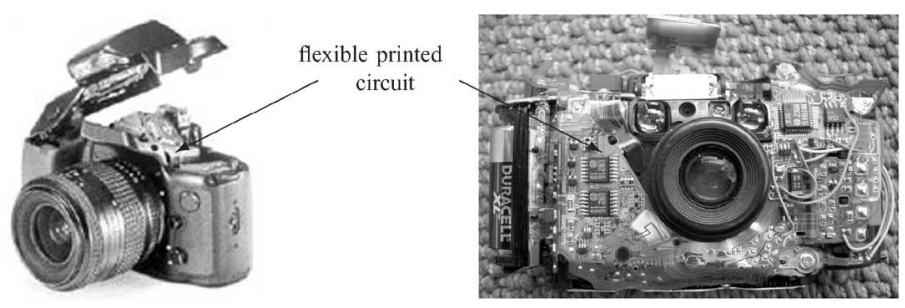

(a)
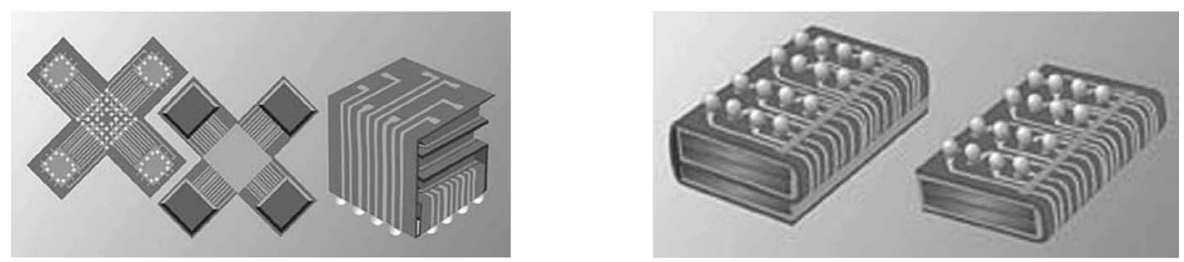

(b)

Fig. 1. Examples of high-density 3-D packaging using PCB technology. (a) Digital photo cameras exploiting flexible printed circuits for complex component placement in 3-D [1]. (b) Encapsulated ICs on flex PCB folded into a surface mountable 3-D package with a small footprint [2].

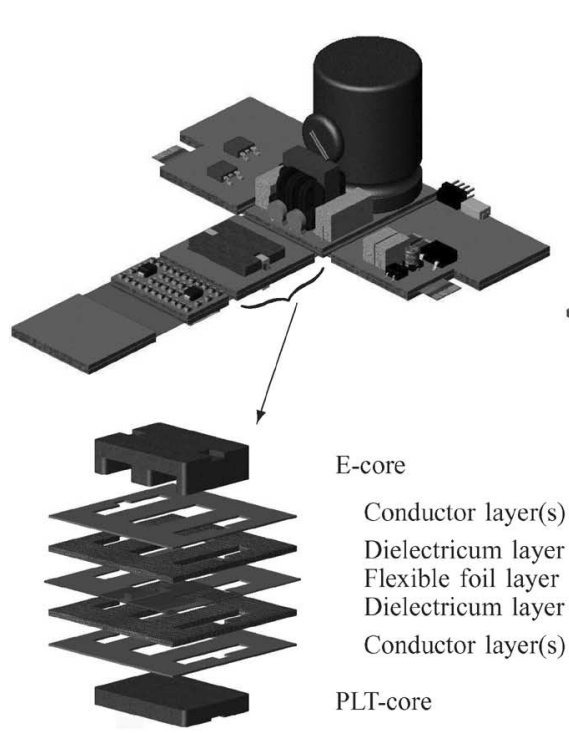

(a)

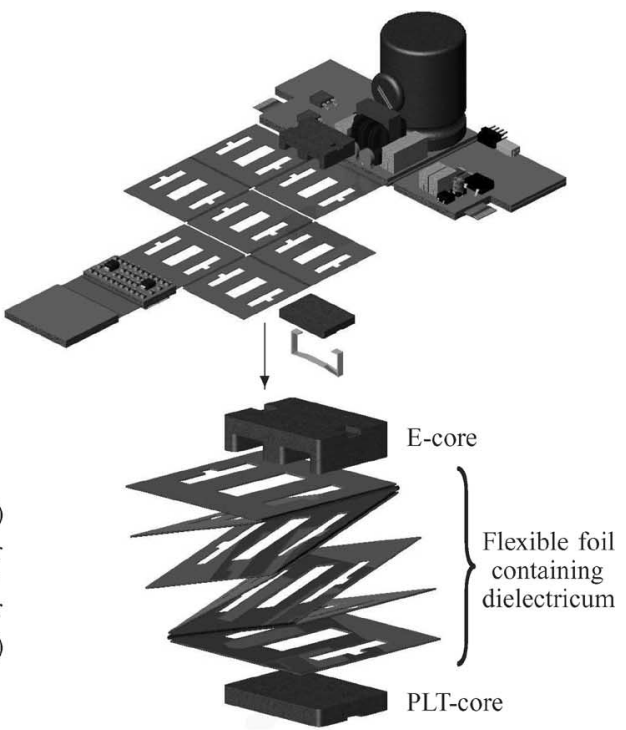

(b)

Fig. 2. Alternative implementations of planar PCB-embedded transformers in power converters. (a) Rigid PCB implementation. (b) Rigid-flex PCB implementation.

transformer windings on respective layers of the PCB that are already present for the rest of the converter [see Fig. 2(a)] [9].

This greatly reduces the number of assembly steps required during manufacturing, but increases the number of layers for the entire PCB to that required by the planar transformer, especially for high-turn-ratio transformers used in off-the-line applications. To counteract this increase of costly layers and subsequent underutilized PCB material, these transformer windings can be formed ("Z"-folded [10], [11]) on a flexible portion of a rigid-flex PCB [see Fig. 2(b)]. This effectively decouples the PCB layers carrying transformer windings from those of the converter with no changes required in manufacturing, only in the artwork design. Techniques originating from the textile industry already exist for the mass production of Z-folded inductors, according to Sippola et al. [12]. The adaptation of such techniques to also include the mass production of Z-folded components that remain fixed to its larger converter system is therefore realistic.

\section{A. PCB Material Saving}

The actual material saving can be seen from Fig. 3. Here, the PCB surface area occupied by the embedded planar transformer is labeled $A_{2}$ and that of the rest of the converter as $A_{1}$, as shown in Fig. 3(a). The PCB surface area required for an $n$-layer embedded planar transformer using a multilayer, rigid (FR-4) PCB is given by

$$
A_{\mathrm{ML}}=n \times\left(A_{1}+A_{2}\right)
$$

whereas that of the rigid-flex solution is given by

$$
A_{\text {flex }}=A_{1}+n \times A_{2} .
$$




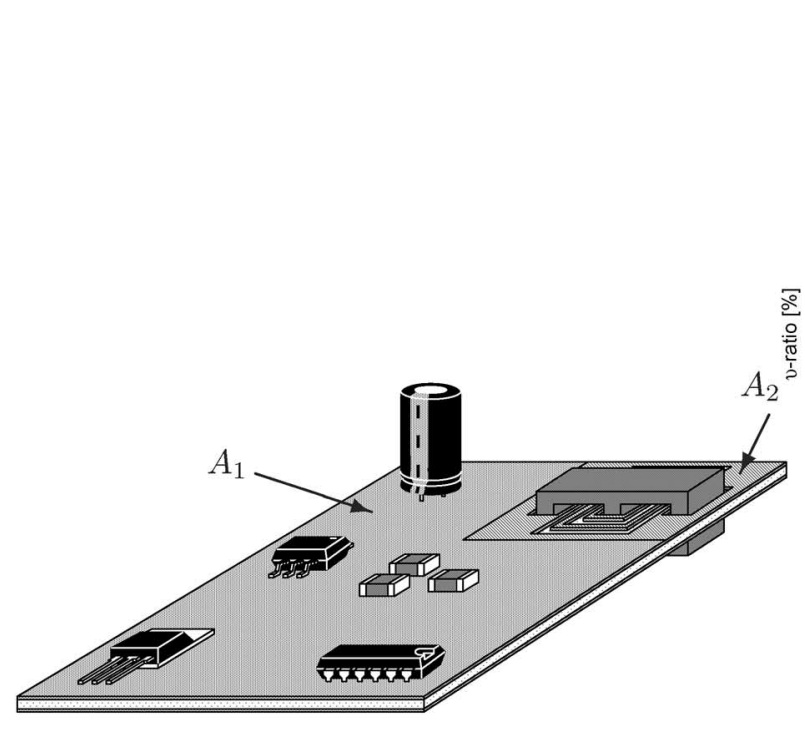

(a)

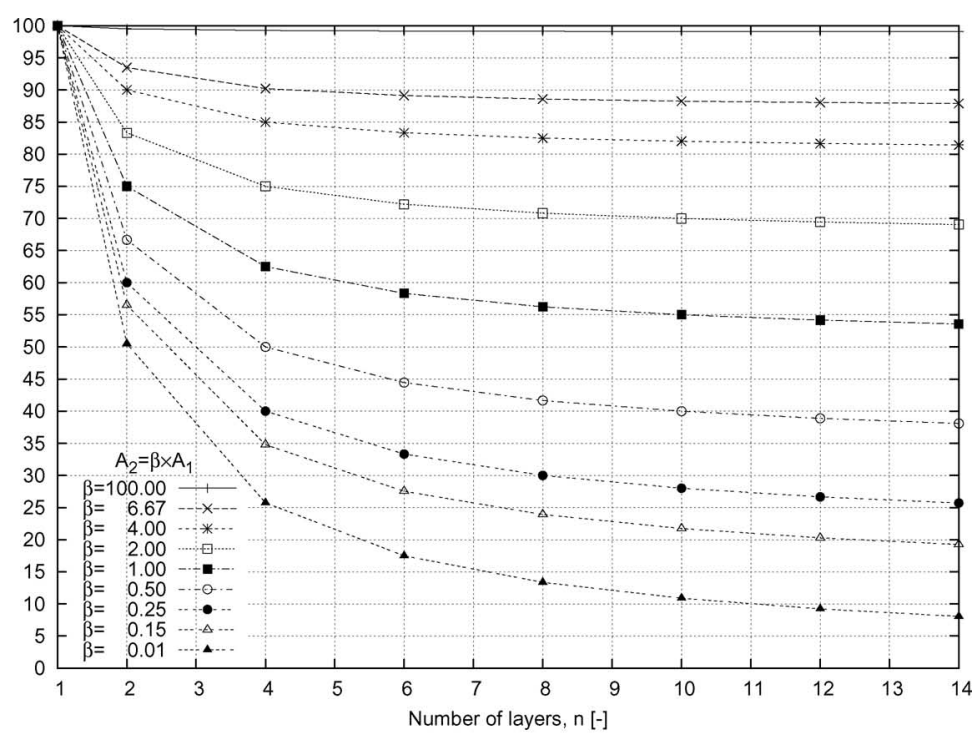

(b)

Fig. 3. Planar embedded transformer example and material usage comparison. (a) PCB-assembled power converter with embedded planar transformer. (b) Required PCB material comparison for various area ratios.

Setting $A_{2}=\beta A_{1}$ and calculating the ratio between these areas for various number of PCB layers results in

$$
v=\frac{A_{\mathrm{flex}}}{A_{\mathrm{ML}}}=\frac{1+n \beta}{n(1+\beta)} .
$$

Fig. 3(b) shows ratio (3) for a variety of $\beta$ ranging from: the transformer surface area is 100 times that of the remaining system $(\beta=100)$, to the PCB surface area for the system is 100 times that of the transformer $(\beta=0.01)$. It can be seen that when the area occupied by the transformer is small compared to the area occupied by the rest of the converter $(\beta \ll 1)$, and the amount of transformer winding layers are substantial (high turn ratio: $n \geq 14$ ), a PCB material saving of more than $80 \%$ is possible when using the rigid-flex PCB planar transformer implementation.

\section{B. PCB Cost}

The number of layers strongly influences the cost of the PCB. This is due to the increased complexity in aligning the layers and keeping them warp-free. The increased complexity in establishing the interlayer metallization (vias) also contributes. An optimistic relation between the amount of layers in a rigid FR-4 PCB and its cost $\left(\operatorname{cost}=n^{1.25}\right)$ is assumed here. ${ }^{2}$ Flexible foils increase the cost as handling and folding very thin substrate material pose new challenges in manufacturing (factor 2 assumed here ${ }^{1,2}$ ). The combination of rigid FR-4 substrates with flexible foils (rigid-flex PCBs) also increases manufacturing cost as the handling, aligning, and folding of rigid PCB in combination with very thin flexible PCB require a lot of expertise (factor 4 assumed here ${ }^{1,2}$ ). Fig. 4 shows how the cost of the three different

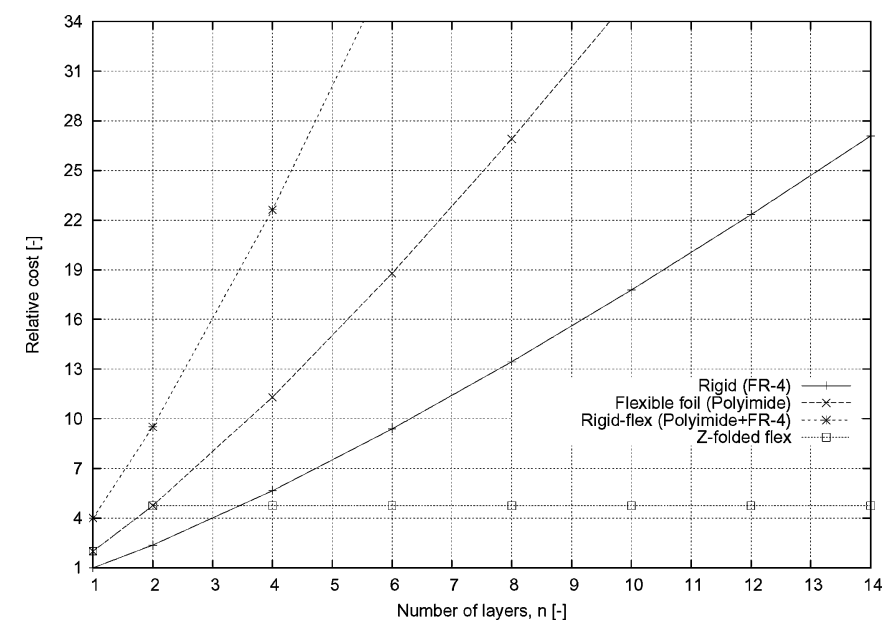

Fig. 4. Relative cost indication for various PCB technologies.

types of PCB structures ${ }^{3}$ compare according to the number of layers. The relative cost of the rigid-flex PCB planar transformer implementation has also been added. It can be seen that the cost saturates approximately at the level of a two-layer flexible-foil solution for all $n>2$, and can be explained by realizing that the additional number of layers are created by means of "Z"-folding and not by adding more PCB material. For this to hold, however, the folding pattern of the "Z"-folded winding structure should also be considered as not every folding pattern makes efficient use of its raw PCB material. Inefficient folding patterns result in a lot of wasted material during manufacturing.

\footnotetext{
${ }^{3}$ In relation to a single-sided, rigid FR-4 PCB.
}

\footnotetext{
${ }^{2}$ This will differ for each individual PCB design as well as per PCB manufacturer as it depends on many technical and economical factors.
} 


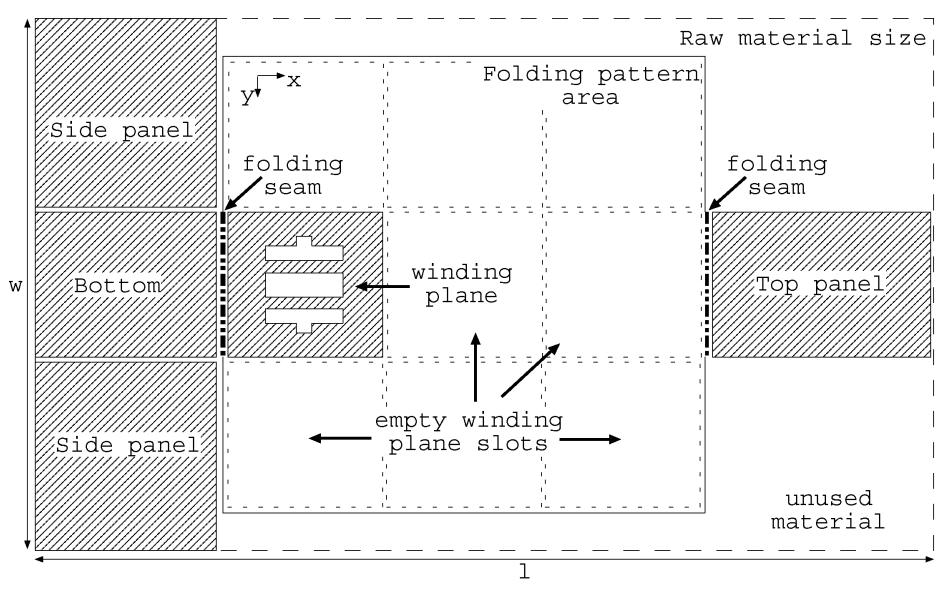

(a)

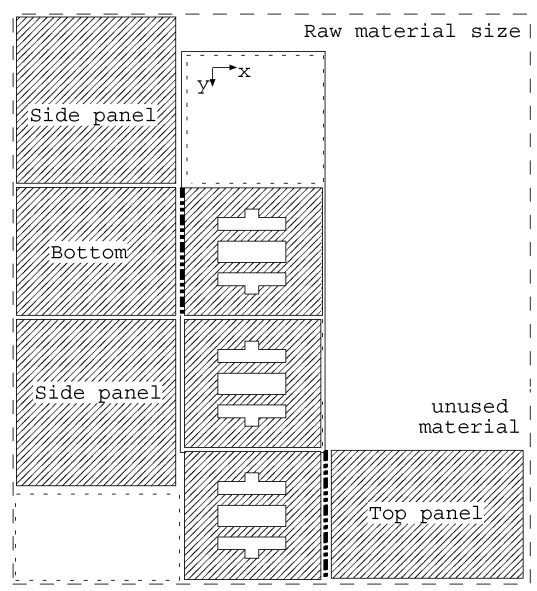

(c)



(b)

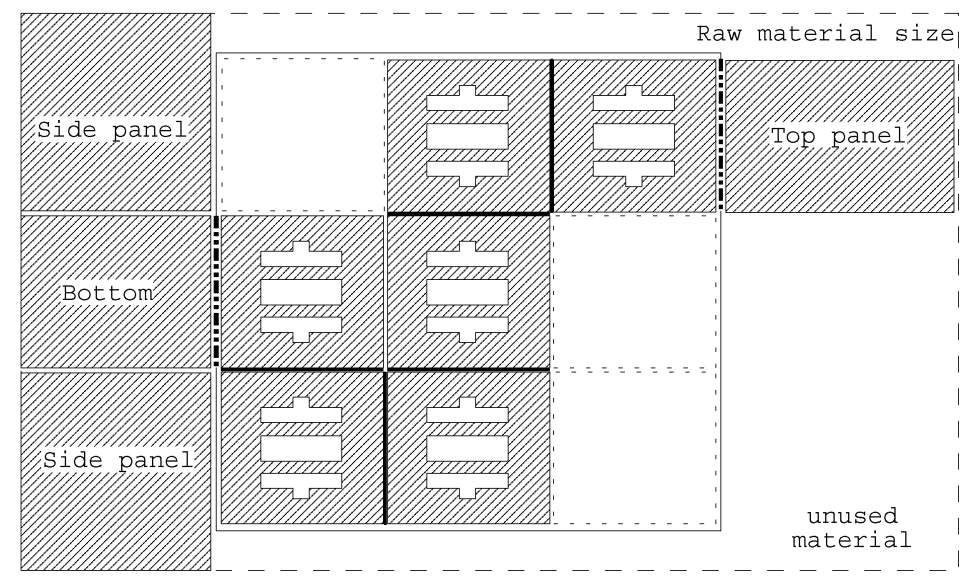

(d)

Fig. 5. Folding pattern definitions and comparative evaluation. (a) Floor plan of rigid-flex PCB converter. (b) Folding pattern comparison on material usage factor. (c) Example A: rigid-flex PCB converter with folding pattern Y3. (d) Example B: rigid-flex PCB converter with folding pattern XY6.

\section{Flexible-Foil Folding Patterns for Transformer Windings}

To investigate the effect which the folding pattern has on the PCB material usage, the rigid-flex PCB converter ${ }^{4}$ portrayed in Fig. 2(b) is revisited. Fig. 5(a) shows the material area of the unfolded PCB showing the rigid-flex side, bottom, and top panels. An integral flexible foil extends from the rigid-flex panel marked "bottom" to form the flexible-foil layers that need to be folded to realize the embedded planar transformer. The folding pattern will define the exact occupation of the "empty winding plane slots" in the "folding pattern area" [see Fig. 5(a)]. After the flexible-foil winding pattern has terminated, the integral flexible foil continues into the adjacent rigid-flex panel marked "top." A PCB material usage factor is then defined as

$$
\begin{aligned}
\tau & =\frac{\text { Area of raw material used }}{\text { Area of raw material }} \\
\tau_{\perp} & =\frac{\tau_{\text {rigid-flex }}}{\tau_{\text {rigid }}}
\end{aligned}
$$

\footnotetext{
${ }^{4}$ Motivation behind the specific layout and PCB buildup in Fig. 2(b) will become clear in Section III.
}

with $\tau_{\perp}$ being the value normalized to layout implementing only the most basic folding pattern (X1 or Y1), therefore not using rigid-flex solution but only the rigid solution.

Folding patterns are conceivable in the main directions in which the winding panels are folded, these are labeled " $\mathrm{X}$ ""Y"-, and "XY"-folding in the $x-, y$-, and both $x$ - and $y$ directions [defined in Fig. 5(a)]. These patterns are shown in Table I and their respective material usage factors compared in Fig. 5(b).

Table I shows only the "folding pattern area" part of the PCB assembly, as indicated in Fig. 5(a), with the folding seam (indicated by the dashed double dotted lines next to the bottom and top panels, respectively) corresponding to those in Table I and indicating where the folded component is attached to the remaining converter assembly. The folding pattern area resembles the PCB material allocated toward creating the folded component. The number of tiles used to create the winding is indicated by "\#” and the picture shows this number of PCB tiles connected to each other by means of the integral layer of flexible PCB (the raw material of the flexible PCB is shown as the solid line around the circumference of the tiles and the empty slots). The folding is performed on the seam between the panels in the direction of the arrow (imagine the arrow in the plane perpendicular to the 
TABLE I

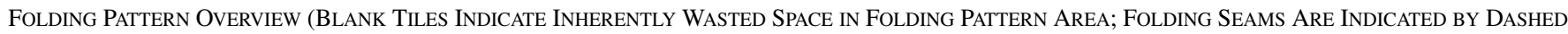
LiNES AND CORRESPOND to THOSE IN FIG. 5, ARrows INDICATE THE PROPOSED Folding PROCEDURE)
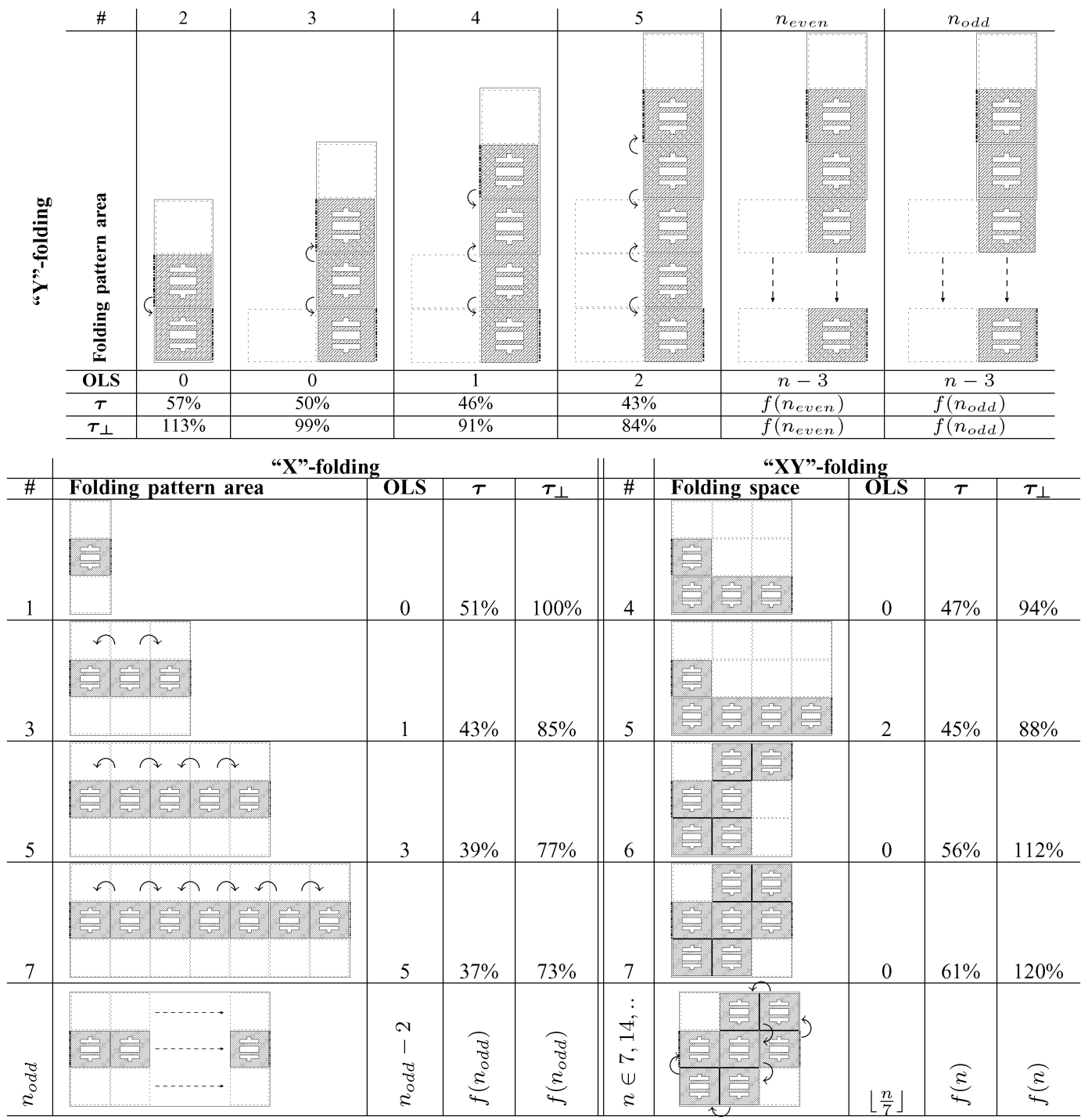

Light dash line: outline of empty winding plane slot.

Light solid line: outline of folding pattern area indicating the width (fixed parameter) of the base case (Y2 pattern) and having variable length. Dashed double dotted line: folding seam from winding plane to connected panels ("Bottom" and "Top panel").

paper, or standing upright). The arrow indicates which panel is folded either into or out of the paper through $180^{\circ}$ and placed on top of the adjacent tile (with the holes for the inductor doubling as alignment markers). For the "X" and "Y" folding patterns the panels are folded on adjacent sides (left and right), but for the "XY" pattern, all sides are folded one after each other (bottom, then left, then top, then right, etc.). For patterns with more than two tiles, the first two tiles (starting either from right or left) are folded, and then the folded tiles can be considered as one tile and the aforementioned folding process can be repeated, taking into account the direction of the arrows, until only one (thick) tile remains.

A unique problem with folding flexible foils to create transformer windings is that the folded seam always turns out thicker ${ }^{5}$ due to an appreciable bending radius required to fold any flexible foil by $180^{\circ}$. This plays a role in the resulting height of the folded winding stack, which has the restriction that it should fit into its magnetic core. Folding patterns that allow seams to be placed at different edges will result in a flatter winding stack than those with all seams on the same edge. The amount of overlapping seams a folding pattern produces is denoted with $O L S$ in Table I.

From Table I, it can be seen that "X"-folding is restricted to only odd number of planes and creates many overlapping seams resulting in a thick winding stack. This can be problematic in

${ }^{5}$ The multilayer PCB embedded transformer does not have this problem but, in turn, requires vias for interlayer metallization where the flex does not. 
combination with the restricted winding window height associated with planar cores. The material usage factor deteriorates sharply [see Fig. 5(b)] due to the size of the raw material expanding in the $x$-direction, and thereby creating more unused raw material next to the folding pattern area [see Fig. 5(a)].

The same holds for the material usage factor when implementing "Y"-folding, as increasing the amount of planes (above 2 in this example) automatically increases the width $(w)$ of the required raw material, unused material is then created next to the side and top panels [see Fig. 5(a)]. Although all number of winding planes are supported by "Y"-folding, the PCB artwork on the top panel is inverted for all even number of winding planes.

"XY"-folding combines folding panels in both the $x$ - and $y$ directions, which results in folding seams being distributed over the four edges evenly producing no overlapping seams (OLS = 0 ), with the exception of five winding planes. This method is only possible with four panels or more if the artwork is not to be inverted on the adjacent PCB panel. It has a material usage factor that increases as the number of panels increase to an optimal value (a multiple of 7 for this example). Being careful not to expand beyond the width of the side panels and keeping the winding panel length $(l)$ to a minimum reduces the amount of wasted material during manufacturing.

The patterns shown in Table I are all serial-connected-type patterns where all winding planes are linked in a head-to-tail configuration. Parallel folding patterns are also possible that utilize all the available pattern area, and therefore have a high material usage factor, but inherently have a high number of overlapping seams due to the many parallel seams that have to be folded-some even folded twice over-to form a single winding stack suitable for a planar core. When a planar core is to be used a folding pattern with high material usage factor $(\tau \gg)$ and low amount of overlapping seams $(\mathrm{OLS} \ll)$ is preferred.

The aforementioned approach leads to a manufacturable embedded planar transformer with optimal PCB material usage. To increase the level of PCB usage in power converters, the functionality of the PCB needs to be investigated further, which is discussed next.

\section{INCREASING PCB FUNCTIONALITY}

Three aspects to increase the functionality of the PCB is considered here: electromagnetic integration of passives, geometrical packaging of bulky, discrete components, and PCB-assisted 3-D thermal management. These are discussed individually next.

\section{A. Integration of Passives}

The electromagnetic integration of passives are steadily becoming feasible for power electronic circuits due to PCB substrate material maturing to realize useful resistance, capacitance, and inductance values [13], [14]. This allows the volume associated with discrete components' packaging to be discarded and higher power densities to be achieved. Two approaches to implementing this level of integration in PCB-assembled power converters can be identified, as illustrated in Fig. 6 for an $L L C$ series-resonant topology, ${ }^{6}$ using the integration convention used by Strydom [16]:

1) electromagnetic integration, using multiple technologies to create a single, electromagnetically integrated [inductor-capacitor-transformer $(L C T)$ ] structure requiring multiple manufacturing processes;

2) technological integration using a single technology platform to create separate, electromagnetically integrated components interconnected by the technology base itself, which are part of one integral manufacturing process.

The multitechnology approach is extensively documented by Strydom et al. [16] and is quite suited for low-voltage-ratio applications such as voltage regulation modules (VRMs), but presents an interesting electromagnetic as well as manufacturing challenge for high-voltage-ratio (off-the-line) applications [17].

The single technology approach is better suited for highvoltage-ratio (off-the-line) applications as will be discussed next. Fig. 7 shows the details in the realization of the flexible-foil PCB that, when folded, creates the transformer winding.

Standard, $1 \mathrm{oz}$. copper, double-sided, polyimide, flexible-foil material is used with the copper plated to $60 \mu \mathrm{m}$ for higher current capability [see Fig. 7(b)]. An "XY"-folding pattern [see Fig. 7(d)] is shown with an appropriate layer of soft magnetic material ${ }^{7}$ [Fig. 7(c)] added in between appropriate flexible PCB winding panels to enhance the leakage inductance in order to use it as a resonant inductor [see Fig. 7(e)]. Furthermore, an air gap in the ferrite core adjusts the magnetizing inductance in a similar way as the multitechnology, single-component $L C T$ approach would [see Fig. 7(f)].

Fig. 8 shows the realization of the half-bridge capacitor structure. The half-bridge capacitors (which also function as a resonant capacitor in this topology) are integrated into the flex foil of an adjacent rigid-flex panel with no changes required in manufacturing, only in the artwork design. The implemented polyimide foil exhibits a dielectric strength of $197 \mathrm{kV} / \mathrm{mm}$ and a relative permittivity of $4\left(\epsilon_{r} \approx 4\right)$. This is sufficient to realize the entire resonant capacitance for rectified line voltage and high-frequency $(>500 \mathrm{kHz})$ operation.

\section{B. Geometrical Packaging}

Another advantage of implementing a flexible PCB in a power converter is the ability to place components more freely in 3-D space. The example shown for microelectronics in Fig. 1 already indicated the volumetric improvement that can be achieved. This can also be applied to power electronics but then the geometrical aspects of the discrete components (diversity and nonuniformity of the component shapes and sizes) need to be considered. The layout and buildup of the rigid-flex PCB converter shown in Fig. 2 have been governed by a geometrical packaging approach introduced in [18]. This approach seeks to identify mutual-compatible component shapes among the remaining discrete converter components that, due to their operating frequency or energy storage function, remain too large to be

\footnotetext{
${ }^{6}$ The topology has been selected based on the integrateability of its passives (resonant tank) as documented in [15].

${ }^{7}$ Ferreira et al. [14] discusses and compares suitable materials for use with PCB technology.
} 


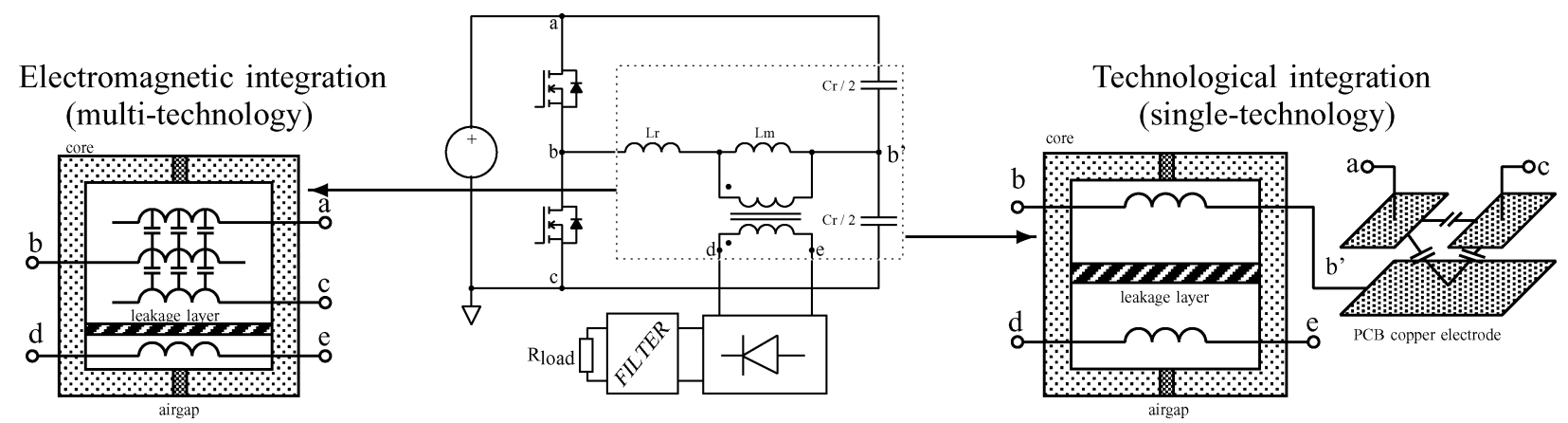

Fig. 6. Integration alternatives: electromagnetic integration in a multitechnology structure, or technological integration into a single technology structure.

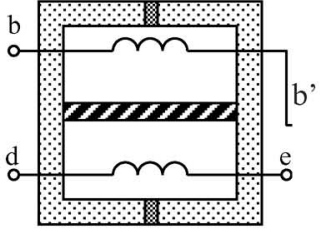

(a)

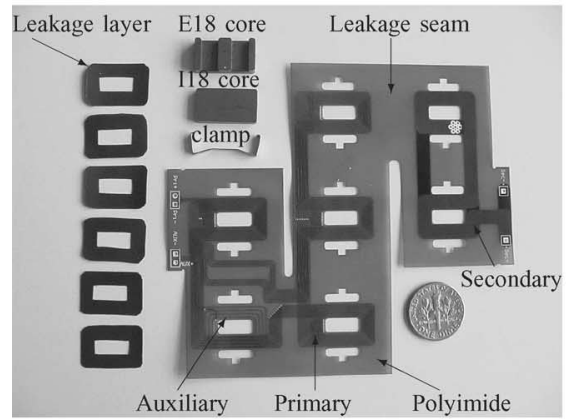

(d)

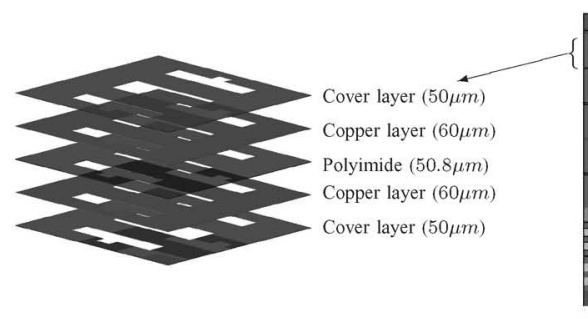

(b)

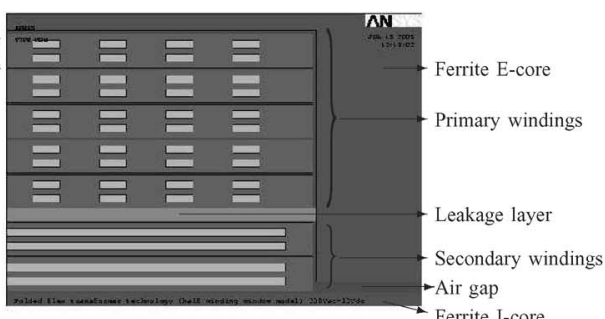

(c)

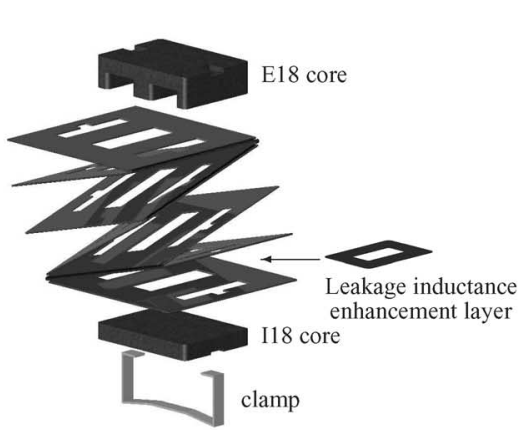

(e)

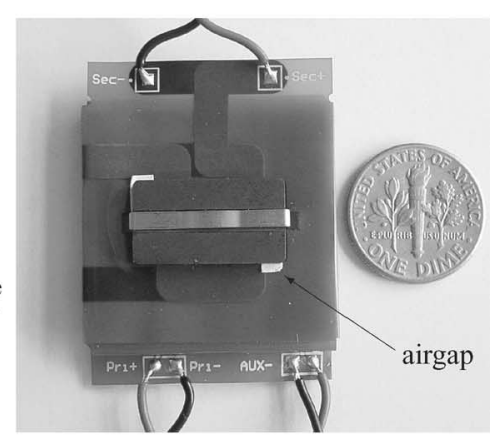

(f)

Fig. 7. Technologically integrated $L L T$ realization using folded, flexible-foil windings. (a) Integrated $L L T$. (b) Flex PCB layer buildup. (c) Cross section (half winding window). (d) Flex PCB, integrated $L L T$ parts. (e) Folding method. (f) Folded flex, integrated $L L T$.

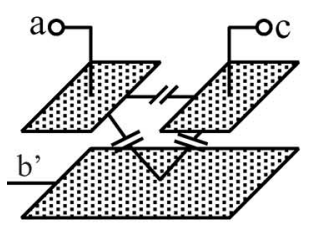

(a)



(b)

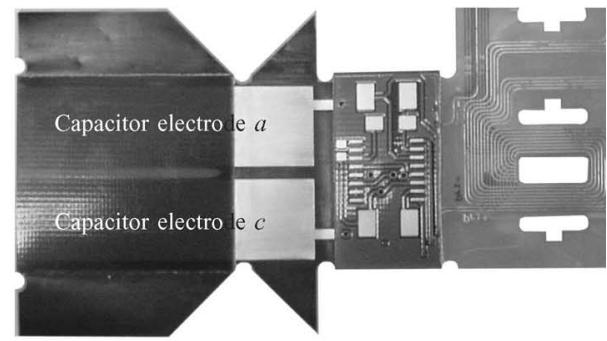

(c)

Fig. 8. Technologically integrated capacitor realization using polyimide flexible printed circuit $\left(\varepsilon_{r} \approx 4\right)$. (a) Integrated C. (b) Outside layer. (c) Inside layer.

integrated into the PCB. The placement is further governed by component-specific temperature guidelines as described in [19]. Rigid-flex PCB technology provides the means to place these components where they complement each other's shape, in order to be packaged in a compact manner while still being electrically interconnected. The principle is shown in Fig. 9. The integral flexible foil (also responsible for realizing the embedded transformer windings) creates hinges on which the rigid panels (on which the discrete components have been strategically placed) can be folded to close the converter in on itself, resulting in a compact converter assembly with very little air enclosed within the converter. 


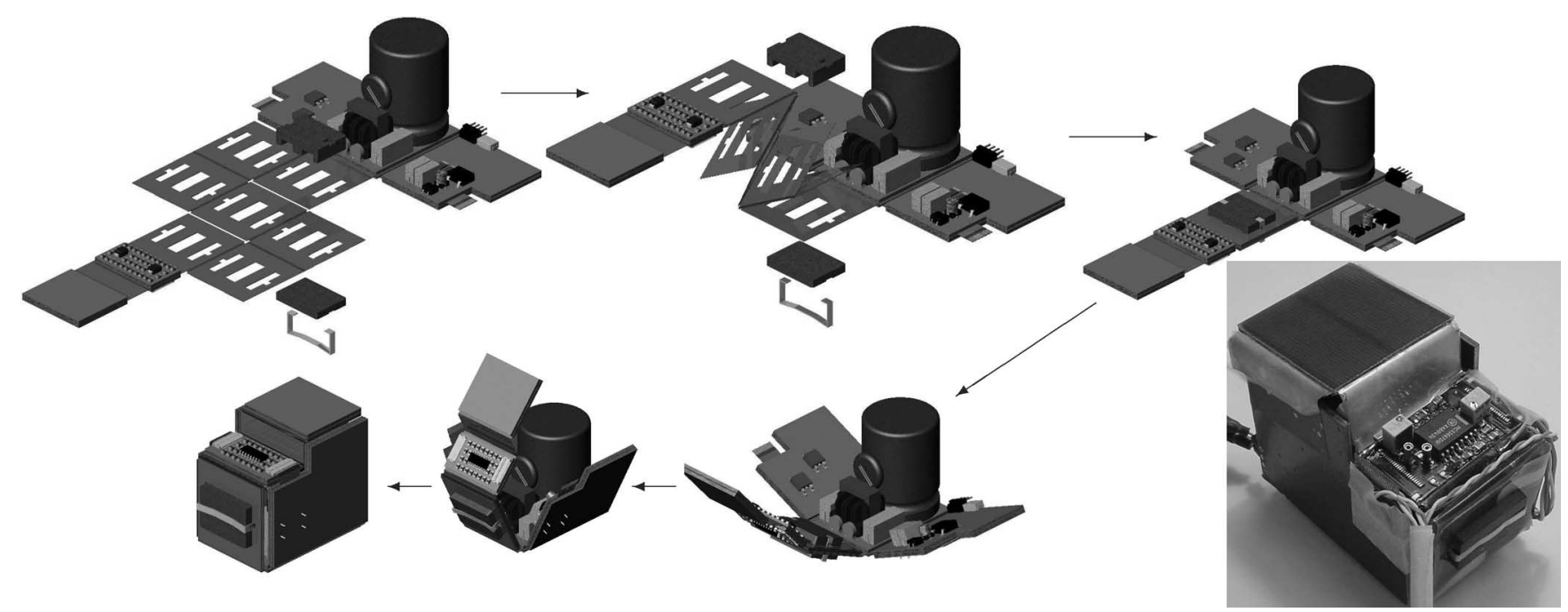

Fig. 9. Geometrical packaging approach implementing rigid-flex PCB with flexible-foil hinges to create a compact, folded 3-D PCB converter.

\section{Thermal Management Enhancement in 3-D}

An inevitable consequence of moving components closer to each other, instigated by geometrical packaging, is the increase of loss density among components and the danger of aggravating the formation of "hot spots" within the fully folded 3-D converter. As with the previous PCB enhancements, the thermal design should now also include the third dimension to be able to do the thermal management of the 3-D converter well. Using the thermal prediction method described in [19], the thermal profile of the unfolded converter assembly can be determined, as shown in Fig. 11. The profile, based on component loss calculations, confirms that the PCB itself takes part in the thermal management to a large extent, thereby increasing its functionality yet again. As an example, all the copper on both the inner flex and outer rigid layers have been retained to act as a heat spreader throughout the converter assembly, a principal that was shown to be very effective in [6]. The effect thereof can already be seen clearly on the spread temperature profile of the integrated transformer structure. This effect is even greater in the folded converter. Furthermore, the outer copper coverage is dedicated to optimal thermal convection to the ambient (eventually through a thin plastic cover when implemented as final consumer product), implying that no electrical connection traces have been routed via these outside layers and that these are thermally coupled to inner layers with an abundance of thermal vias. Thermal vias have been used extensively below known heat sources, such as the power semiconductor devices, to incorporate as much of the PCB copper as heat spreader and reduce the thermal resistance from the component surface to the ultimate convection surface. Components have been placed in the converter design keeping the respective loss dissipation in mind. Therefore, the one side panel is dedicated to the power switches and the other side panel to the output rectifier with its power diode bridge. In general, the greater the expected loss dissipation for a specific component, the more copper coverage is allocated to that components thermal management. Thermal interface material between components helps reduce the thermal resistance from within the core of the converter assembly to the outside convection surface.

\section{CONCEPT Evaluation}

To illustrate the next level of PCB usage in power electronic converters, the following technology demonstrator has been synthesized according to the approach detailed in Section II and III. It will be evaluated quantitatively and critically here.

\section{A. Technology Demonstrator}

A $20-\mathrm{W}, 220-\mathrm{V}_{\mathrm{ac}}-12-\mathrm{V}_{\mathrm{dc}}$ (off-the-line) converter has been developed based on the series LLC topology (Fig. 6, middle) operating at $530 \mathrm{kHz}$. Careful circuit design reveals integrateable values for the resonant tank being $L_{m} \approx 300 \mu \mathrm{H}$, $L_{r} \approx 100 \mu \mathrm{H}$, and $C_{r} \approx 780 \mathrm{pF}$. The respective inductances are obtained by 34 primary windings $(2 \times 200 \mu \mathrm{m}$ wide $)$ on a planar core (EI18, 3F4 material) with an effective core air gap of $200 \mu \mathrm{m}$ and a 700- $\mu \mathrm{m}$ leakage layer, constructed from ferrite polymer composite (FPC) material introduced between the primary and secondary windings [Fig. 7(e)]. These 34 primary windings are spread over five double-sided, flexible-foil panels and " $Z$ "-folded to form the continuous winding stack implementing a "XY"-folding pattern (see Fig. 7 and Section II-C). Using the single technology integration approach presented in Section III-A, the integrated capacitors are implemented in the series connected, three-level configuration in an adjacent panel of the flex PCB, as shown in Fig. 8. This has the advantage that the parasitic capacitor formed between terminals a and c (although small) contributes toward the bus capacitance.

Combining the planar PCB-embedded transformer (Fig. 7) with the integrated flexible-foil PCB capacitors (Fig. 8) into the rigid-flex PCB structure culminates to the geometrically packaged 3-D PCB converter showcasing the next level of PCB usage, as shown in Fig. 10. The single PCB manufacturing process using inherently compatible materials simplifies the converter assembly considerably and even removes a few sources of converter failure (incompatible coefficients of expansion and winding interconnections) allowing for increased reliability. 


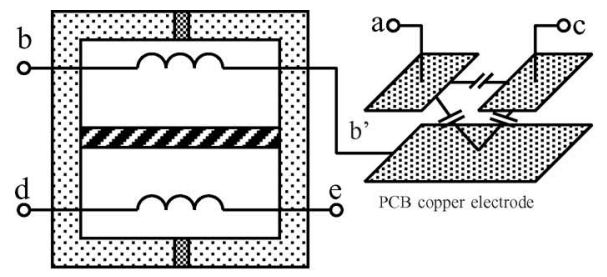

(a)

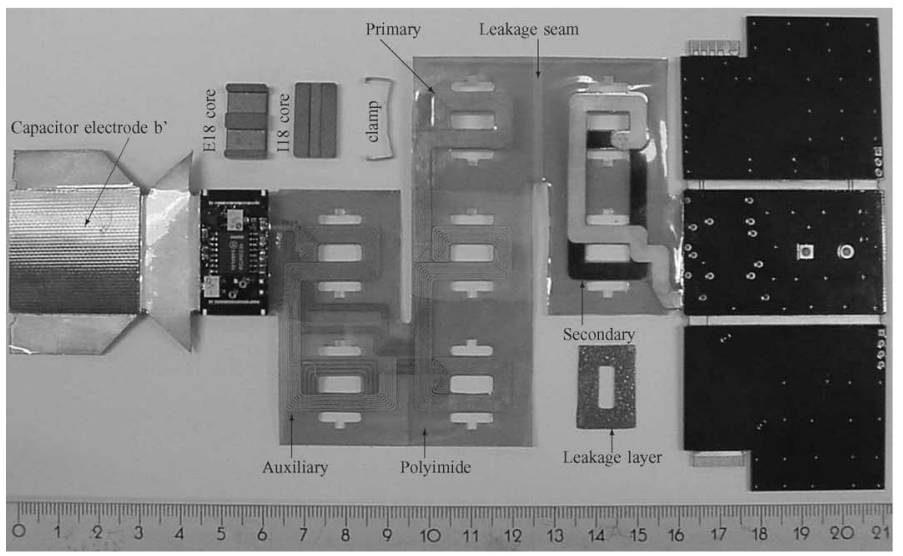

(b)

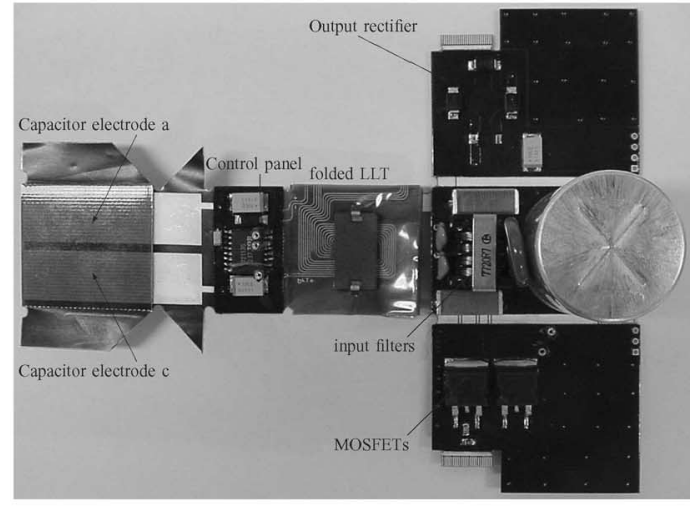

(c)

Fig. 10. Geometrically packaged 3-D PCB converter showcasing the next level of PCB usage (PCB-embedded transformer and flexible-foil PCB capacitors). (a) Multifunctional, single technology, PCB integrated components. (b) Unfolded rigid-flex 3-D PCB converter showing integrated parts. (c) Rigid-flex 3-D PCB converter with fully folded transformer.

\section{B. Thermal Testing}

An extended full-power test $(20 \mathrm{~W})$ has been performed on the 3-D PCB converter. The resulting thermal profiles ${ }^{8}$ are shown in Figs. 11 and 12. The predicted surface temperature profile ${ }^{9}$ [Fig. 11(c)] is validated by the close correlation with the measured surface temperature profile of the actual unfolded 3-D PCB converter [Fig. 11(d)], measured under thermal steadystate, full-output power conditions.

The thermal management aspects incorporated in the folded 3-D PCB, shown in Figs. 12(a) and 13, include uninterrupted copper coverage on the inner flexible printed circuit as well as the rigid panels, to facilitate the most efficient heat spreading on the outer surface of the folded converter. The copper-covered flexible foil hinges create a low thermal resistance connection from the bottom to the side panels, and the thermal flaps on the top panels do the same for the top-to-side connections. Thermal vias reduce the thermal resistance between the four stacked PCB layers as well as from high dissipation components, such as the two half-bridge transistors. Interface material between components inside the folded structure and the PCB itself, such as the capacitor canister, further enhances the thermal pathways throughout the system.

During the thermal measurements, the IR thermography is assisted by strategically placed thermocouples placed within the folded structure. This enables the monitoring of the vital component temperatures hidden from the IR camera view. The

\footnotetext{
${ }^{8}$ Measured using IR thermography.

${ }^{9}$ Based on a finite difference method (FDM) estimation approach introduced in [18].
}

measured surface temperatures and profile of the outer surface are shown in Fig. 12(c) and (d), respectively, with the inner component temperatures at the same instant shown alongside Fig. 12(b). The enhanced heat-spreading capability of the 3-D PCB converter, once folded, is evident from these measurements. The highest surface temperature on any outer surface of the converter is measured to be $52^{\circ} \mathrm{C}$, with an all-round average surface temperature closer to $45^{\circ} \mathrm{C}$.

\section{Impact on System Performance}

In order to assess the impact on system performance as well as manufacturing, brought about when evolving to the next level of PCB usage in power converters, the performance indicators (figures of merit) for determining level of integration as well as thermal management are used for benchmarking, as described by Popović and Ferreira [20] and de Jong et al. [21], respectively. These performance indicators are as follows.

1) Functional elements integration level $\left(K_{I}\right)$ is defined as

$$
K_{I}=\frac{\sum_{i} N_{\mathrm{FE} v_{i}}}{N_{\mathrm{FE}}}=\frac{N_{\mathrm{FE} v}}{N_{\mathrm{FE}}}
$$

where $N_{\mathrm{FE}}$ is the total number of functional elements in the assembly, $N_{\mathrm{FE} v_{i}}$ is the number of virtual functional elements that the functional element $i$ is worth, and $N_{\mathrm{FE} v}$ is the total number of virtual functional elements in the converter. For more details, see [20]. 


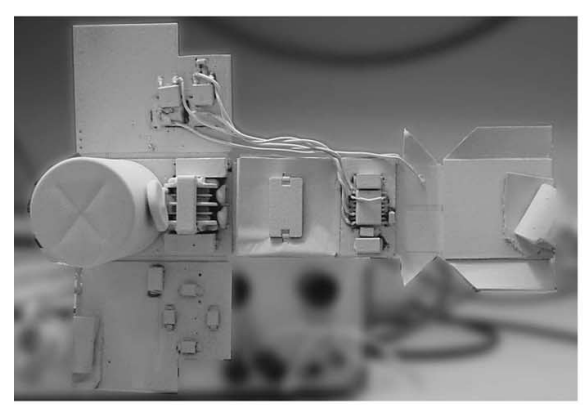

(a)

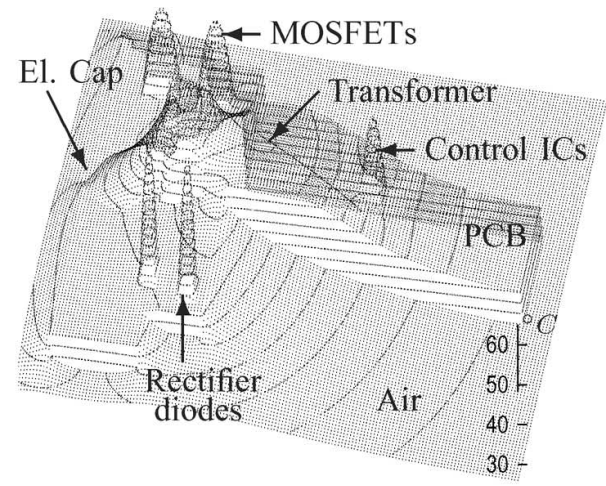

(c)

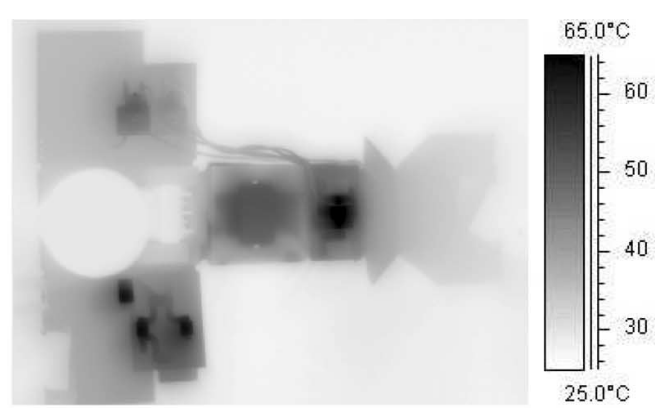

(b)

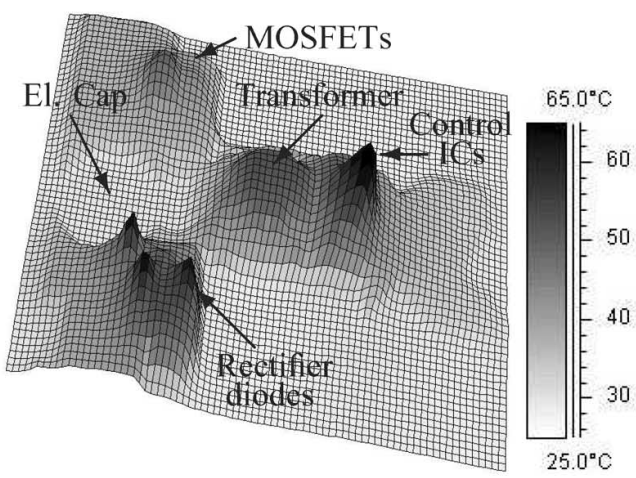

(d)

Fig. 11. Thermal profile of the unfolded 3-D PCB converter. (a) Unfolded 3-D PCB converter. (b) Surface temperatures. (c) Predicted surface temperature profile. (d) Measured surface temperature profile.

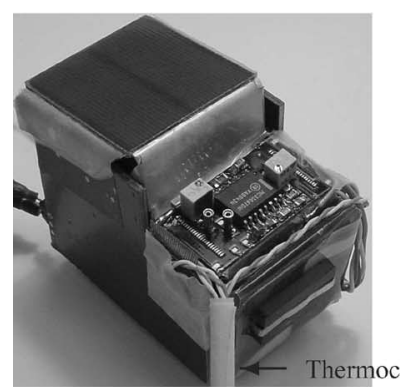

(a)

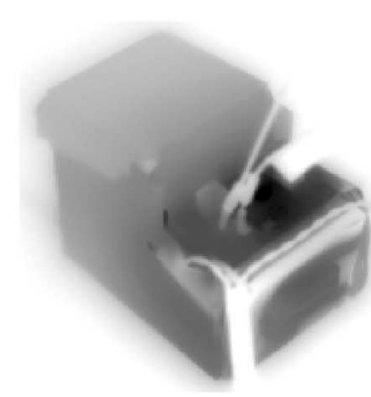

(c)

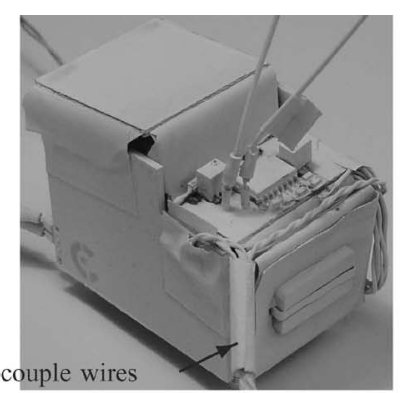

(b)

\begin{tabular}{|l|l|}
\hline \multicolumn{2}{|c|}{ Thermocouple measurements } \\
\hline Gate driver & $84.0^{\circ} \mathrm{C}$ \\
\hline Output diode $\left(D_{1}\right)$ & $71.1^{\circ} \mathrm{C}$ \\
\hline Top MOSFET $\left(S_{1}\right)$ & $50.8^{\circ} \mathrm{C}$ \\
\hline
\end{tabular}
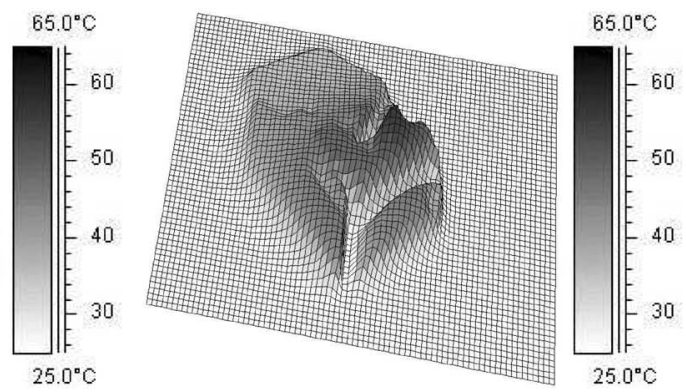

(d)

Fig. 12. Thermal profile of the fully folded 3-D PCB converter. (a) Folded 3-D PCB converter. (b) Monochromatic folded 3-D PCB converter during measurement. (c) Surface temperatures. (d) Surface temperature profile.

2) Packaging elements integration level $\left(K_{P}\right)$ is defined as

$$
K_{P}=\frac{\sum_{j} N_{\mathrm{PE} v_{j}}}{N_{\mathrm{PE}}}=\frac{N_{\mathrm{PE} v}}{N_{\mathrm{PE}}}
$$

where $N_{\mathrm{PE}}$ is the total number of packaging elements while $N_{\mathrm{PE} v}$ is the total number of virtual packaging elements in the subcircuit. For more details, see [20].

3) Thermal management loss density (TMLD) is defined as the ratio between the electrical losses that need to be 
TABLE II

PERFORMANCE INDICATOR OVERVIEW

\begin{tabular}{|c|c|c|c|c|}
\hline \multicolumn{5}{|c|}{ Integration [20], [22] } \\
\hline Parameter & Formula & Description & Conventional & Improved \\
\hline$K_{I}$ & $\frac{N_{F E v}}{N_{F E}}$ & Functional element integration level [-] & 1.00 & 1.46 \\
\hline$K_{P}$ & $\frac{N_{P E v}}{N_{P E}}$ & Packaging element integration level [-] & 1.55 & 1.66 \\
\hline$\eta_{v}$ & $\frac{V_{F E}}{V_{T O T}}$ & Volumetric packaging effectiveness [\%] & 14.67 & 37.62 \\
\hline \multicolumn{5}{|c|}{ Thermal management [21] } \\
\hline Parameter & Formula & Description & Conventional & Improved \\
\hline$T M L D$ & $\frac{P_{\text {losses }}}{V_{T M}}$ & Thermal management loss density $\left[\mu \mathrm{W} / \mathrm{mm}^{3}\right]$ & 95.85 & 1201.52 \\
\hline$T D R_{P D}$ & $\exp \left[-4\left(\frac{T-T_{\text {optimal }}}{T_{\max }-T_{\text {optimal }}}\right)^{2}\right]$ & $\begin{array}{l}\text { Thermal design rating-power density objective }(\alpha=1 \%)[\%] \\
\text { Thermal design rating-power density objective }(\alpha=50 \%)[\%] \\
\text { Thermal design rating-power density objective }(\alpha=85 \%)[\%]\end{array}$ & $\begin{array}{r}29.0 \\
19.35 \\
16.13\end{array}$ & $\begin{array}{l}45.16 \\
29.03 \\
16.13\end{array}$ \\
\hline$T D R_{R}$ & $\exp \left[-4\left(\frac{T-T_{\text {optimal }}}{T_{\max }-T_{\text {optimal }}}\right)^{2}\right]$ & $\begin{array}{l}\text { Thermal design rating-reliability objective }(\alpha=1 \%)[\%] \\
\text { Thermal design rating-reliability objective }(\alpha=50 \%)[\%] \\
\text { Thermal design rating-reliability objective }(\alpha=85 \%)[\%]\end{array}$ & $\begin{array}{c}96.77 \\
49.33 \\
3.2\end{array}$ & $\begin{array}{r}100 \\
90.32 \\
70.97\end{array}$ \\
\hline \multicolumn{5}{|c|}{ Power density } \\
\hline Parameter & Formula & Description & Conventional & Improved \\
\hline$\eta$ & $\frac{P_{\text {out }}}{V_{\text {TOT }}}$ & Power density $[W / l]$ & $\approx 150$ & $\approx 250$ \\
\hline
\end{tabular}

removed from a system and the volume of the thermal management material that needs to transport the heat caused by these losses, and is given by

$$
\mathrm{TMLD}=\frac{P_{\text {losses }}}{V_{\mathrm{TM}}}
$$

measured in watts per cubic meter. $P_{\text {losses }}$ represents the total power dissipation $V_{\mathrm{TM}} . V_{\mathrm{TM}}$ represents the volume of the thermal management material. For more details, see [21].

The outcome of these performance indicators as applied to the rigid-flex 3-D PCB converter is shown in Table II. From Table II, the increase in level of integration, brought about by the integration of the $L L T$ and resonant capacitor components into the PCB as well as implementing the PCB as thermal management, is clearly visible from both the functional element and packaging element integration level indicators. The functional element integration level has increased from 1.00, in the discrete converter realization built on conventional technology, to 1.46 in the 3-D PCB converter. This is due to the integration of functional elements into the PCB such as conductive functions in the form of transformer windings as PCB traces, thermal functions of the heat sink in the form of copper planes and thermal vias, and capacitive and inductive functions of the resonant tank components in the integrated $L L T$ and integrated resonant capacitor structures. The packaging elements integration level has also increased from 1.51 to 1.66 as a result of existing packaging material being assigned more functionality, such as the PCB now performing protection of the inner circuit, thermal management, and electromagnetic interference (EMI) shielding through thick copper planes on top of its basic electrical interconnection, mechanical support, and insulation functionality, among others. These implemented integration efforts have helped increase the volumetric packaging effectiveness-defined as the ratio between volume occupied by functional element material and the total converter volume-from $14.67 \%$ for the discrete converter to $37.62 \%$ for the 3-D PCB converter. This shows that the integration technology proposed herein enables a productive volume utilization of approximately $40 \%$, reducing the overhead of material by approximately $25 \%$ from the conventional discrete converter.

The TMLD value, shown in Table II, indicates a strong increase in the utilization of PCB as thermal management material, resulting in a more uniform temperature distribution along the outer surface of the 3-D PCB converter. It implements its thermal management at $1201.52 \mu \mathrm{W} / \mathrm{mm}^{3}$. The intensified use of available material for thermal management can be seen in the measures implemented in the 3-D PCB: thermal vias, thick uninterrupted copper planes, thermal connections (flaps) to connect external heat spreaders when folded, and thermal interface material between components, as highlighted in Fig. 13.

The thermal design rating $\left(\mathrm{TDR}_{\mathrm{PD}}\right)$ values, shown in Table II, indicate that $\approx 10 \%$ more components operate in a set optimal band at $50 \%$ of its optimal temperature in the 3-D PCB converter than was the case in the conventional discrete converter, when applying the predefined power density objective set, as defined in [18]. Sixteen percent of all the converter components operate above $85 \%$ of their individual optimal temperatures, indicating that the thermal management is not only keeping the components from thermal destruction but also managing the local component temperatures in such a way that their individual operating temperatures approach their optimal temperatures better, resulting in increased profit from the components performance. Ideally, these values should be still higher so that component specifications can be reduced or perhaps cheaper component alternatives implemented, but 3-D layout and material limitations often inhibit the freedom to thermally design to such precision, as is the case for the technology demonstrator here.

The 3-D PCB converter excels when the predefined reliability objective set [18] is applied $\left(\mathrm{TDR}_{R}\right)$. It has an astounding $\approx 71 \%$ of all its components operating above $85 \%$ of their optimal temperature. Ninety percent of its components operate above $50 \%$ of their optimal temperature while the conventional 2-D converter only has $\approx 49 \%$ of its components operating in that 


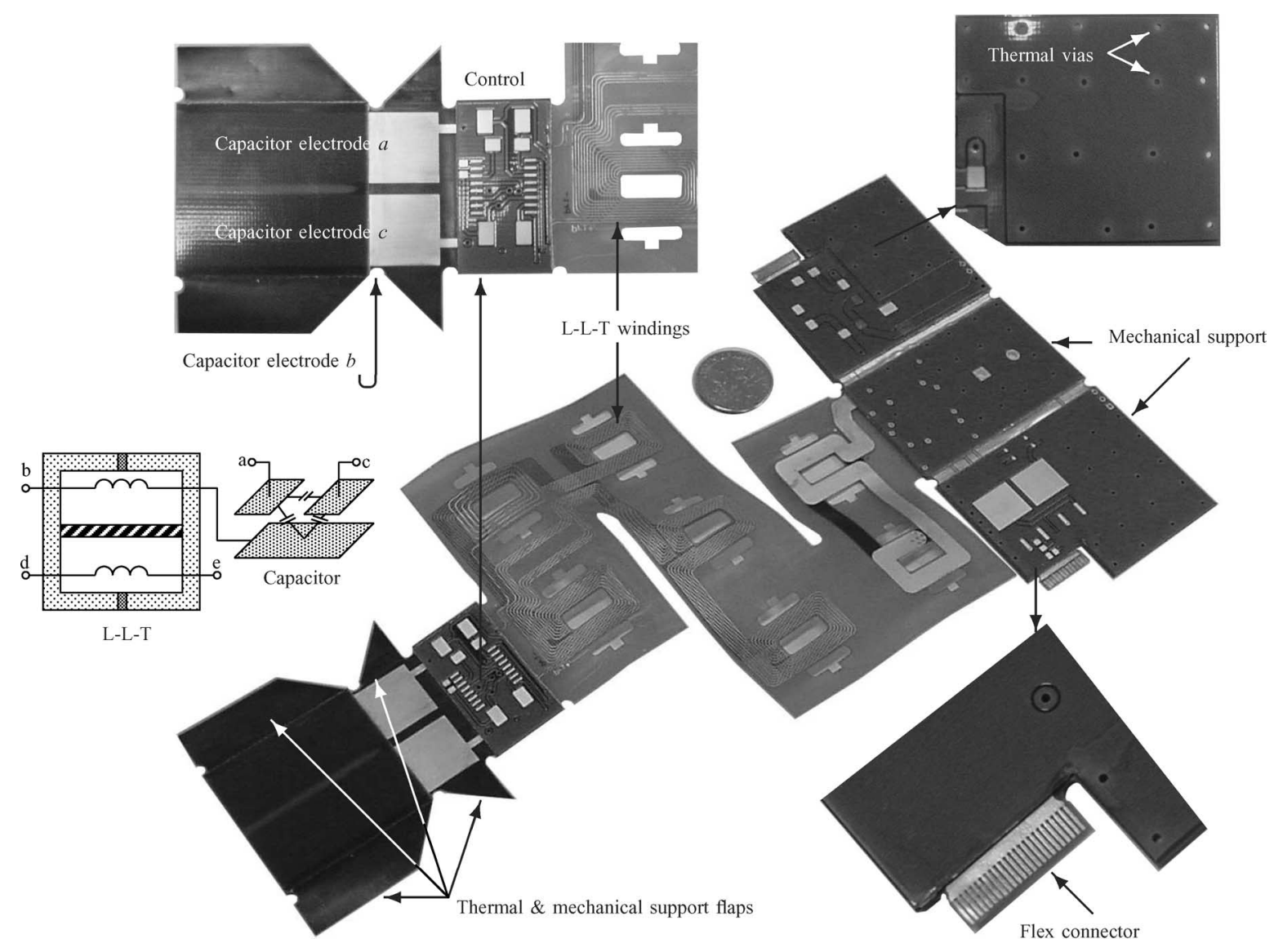

Fig. 13. Overview of the increased functionality of the PCB.

region. A power density improvement from $150 \mathrm{~W} / \mathrm{L}\left(2.5 \mathrm{~W} / \mathrm{in}^{3}\right)$ to $250 \mathrm{~W} / \mathrm{L}\left(4.1 \mathrm{~W} / \mathrm{in}^{3}\right)$ is achieved.

The drawbacks encountered include the following two aspects: the formation of microcracks in the conductor traces of the integrated magnetic structure when the flexible PCB material is folded to create the transformer winding spiral. These microcracks could in a worst case situation increase the trace resistance, and thereby degrade the transformers electromagnetic performance, electrical interconnection failure occurring between traces terminating into microvias located close to the edge of a rigid PCB board or relatively close to a folding seam. The forces acting on the extremely small copper connection between the microvia and the thin conductor trace during the folding procedure are sufficient to physically break its connection if it is located too close to a folding seam or if the flexible PCB is folded and unfolded repetitively. This is a severe problem during prototyping but is unlikely to hinder the actual product reliability as the folding procedure will eventually be performed only once during the automatic manufacturing process.

\section{CONCLUSION}

The PCB usage in power converters has been investigated and improvements to achieve the next level thereof explored. In this regard, the implementation of an embedded planar transformer as high-frequency power transformer in a PCBassembled power converter has been revisited. It has been shown that material and manufacturing cost can be reduced substantially by including flexible-foil PCB into the otherwise rigid PCB structure to create the many winding layers required for a high-turn-ratio transformer assembly without increasing the number of (expensive) rigid PCB layers of the remaining converter. The aspects concerning the successful implementation of the transformer, such as the associated folding pattern of the flexible-foil PCB and its effect on effective PCB material usage, received careful, quantitative attention. Furthermore, increasing of PCB functionality has been addressed in three main topics: integration of passives, geometrical packaging, and 3-D thermal management enhancement. It has been shown that it is possible to integrate high-frequency passives straight into the flexible-foil PCB that is also responsible for creating the embedded planar transformer while the entire converter is being geometrically packaged in 3-D to find mutual-compatible component shapes amongst the bulky, low-frequency (line filter) components to create a power-dense converter. The technology demonstrator's power density has been improved from $150 \mathrm{~W} / \mathrm{L}\left(2.5 \mathrm{~W} / \mathrm{in}^{3}\right)$ to $250 \mathrm{~W} / \mathrm{L}\left(4.1 \mathrm{~W} / \mathrm{in}^{3}\right)$. The PCBs functionality is then extended to include the thermal management of the compact 3-D converter. The PCBs role in creating a thermal path between the densely packed components in order for each individual component to operate at its optimal operating temperature as well as create a uniform temperature distribution on the outer surface of the converter PCB is quantitatively governed by thermal management and integration performance indicators. An integrated 
3-D PCB converter has been synthesized, quantitatively verified, and critically discussed in order to ascertain whether the proposed steps are indeed the ones required to reach the next level of PCB usage in power electronic converters.

\section{REFERENCES}

[1] Flexible Printed Circuits Open Up a Multitude of Innovative Applications, Freudenberg Mektec Europa GmbH, PCB007, Erkelenz, Germany, Mar. 2004.

[2] J. Fjelstad. (2001, Jan.). Flexible circuit origami: The art of packaging IC chips with flexible circuits. CircuiTree [Online]. Available: http://www.circuitree.com/Articles/Column/f3faa14b3d7d7010VgnVCM $100000 \mathrm{f} 932 \mathrm{a} 8 \mathrm{c} 0$.

[3] A. Lostetter, F. Barlow, A. Elshabini, K. Olejniczak, and S. Ang, "Polymer thick film (PTF) and flex technologies for low cost power electronics packaging," in Proc. Int. Workshop Integr. Power Packag. (IWIPP), Jul. 2000, vol. 1, pp. 33-40.

[4] E. Waffenschmidt and J. Ferreira, "Embedded passives integrated circuits for power converters," in Proc. IEEE Power Electron. Spec. Conf., Jun. 2002, vol. 1, pp. 12-17.

[5] W. Brown, A. Malshe, T. Railkar, T. Lenihan, J. Stone, W. Sommers, and L. Schaper, "Thermal management issues and evaluation of a novel, flexible substrate, 3-dimensional (3-D) packaging concept," in Proc. IEEE Int. Conf. Multichip Modules High Density Packag., Denver, CO, Apr. 1517, 1998, pp. 135-140.

[6] E. de Jong, J. Ferreira, and P. Bauer, "Improving the thermal management of AC-DC converters using integration technologies," in Proc. IEEE Ind. Appl. Conf. Oct. 3-7, 2004, vol. 4, pp. 2315-2322.

[7] D. Grafham. (1998, Oct. 12). Optimal thermal management of planar magnetics in high frequency SMPS [Online]. Available: www.paytongroup. com

[8] S. Ben Yaakov. (2001). The benefits of planar magnetics in HF power conversion [Online]. Available: www.paytongroup.com

[9] J. Marinos. (2000, Aug.). Designing planar magnetics [Online]. Available: www.pcim.com

[10] P. Zumel, R. Prieto, J. Cobos, and J. Uceda, "Comparative study of flexfoil technology in HF planar transformer windings," in Proc. IEEE Power Electron. Spec. Conf., Jun. 2002, vol. 3, pp. 1248-1253.

[11] K. Ngo, R. Alley, and A. Yerman, "Fabrication method for a winding assembly with a large number of planar layers," IEEE Trans. Power Electron., vol. 8, no. 1, pp. 55-61, Jan. 1993.

[12] M. Sippola, E. Oyj, and F. Bescherer, "Reel-to-reel manufacturable, leadless surface mountable, z-folded planar power transformers," in Proc. Int. Conf. Integr. Power Syst., Verband der Elektrotechnik (VDE), Jun. 2006, vol. 1 , pp. 255-260.

[13] E. Waffenschmidt, B. Ackermann, and J. Ferreira, "Design method and material technologies for passives in printed circuit board embedded circuits," IEEE Trans. Power Electron., vol. 20, no. 3, pp. 576-584, May 2005.

[14] J. Ferreira, E. Waffenschmidt, J. Strydom, and J. van Wyk, "Embedded capacitance in the PCB of switchmode converters," in Proc. IEEE Power Electron. Spec. Conf., Jun. 2002, vol. 1, pp. 119-123.

[15] E. de Jong, J. Ferreira, and P. Bauer, "LCT integration optimization on a printed circuit board technology platform," in Proc. IEEE Ind. Appl. Conf., Tampa, FL, Oct. 8-12, 2006, vol. 4, pp. 1849-1856.

[16] J. Strydom, "Electromagnetic design of integrated resonatortransformers," Ph.D. dissertation, Rand Afrikaans Univ., Johannesburg, South Africa, Dec. 2001.

[17] E. de Jong, J. Ferreira, and P. Bauer, "Integrated flex winding realisation for 3D PCB converters," in Proc. IEEE Power Electron. Spec. Conf., Jun. 2006, vol. 1, pp. 2878-2884.

[18] E. de Jong, J. Ferreira, and P. Bauer, "Design techniques for thermal management in switch mode converters," IEEE Trans. Ind. Appl., vol. 42, no. 4, pp. 1375-1386, Nov./Dec. 2006.

[19] E. de Jong, J. Ferreira, and P. Bauer, "Thermal model extraction as means to thermal management improvement in PCB assembled power converters," in Proc. Power Convers. Intell. Motion (PCIM) Conf., Jun. 2005, vol. 1, pp. 240-245.

[20] J. Popović and J. Ferreira, "An approach to deal with packaging in power electronics," IEEE Trans. Power Electron., vol. 20, no. 3, pp. 550-557, May 2005.

[21] E. de Jong, J. Ferreira, and P. Bauer, "Evaluating Thermal Management Efficiency in Converters," in Proc. IEEE Power Electron. Spec. Conf. Jun. 20-25, 2004, vol. 6, pp. 4881-4887.
[22] J. Popović and J. Ferreira, "Converter concepts to increase the integration level," IEEE Trans. Power Electron., vol. 20, no. 3, pp. 558-565, May 2005.

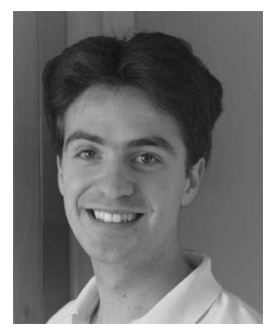

Erik C. W. de Jong (S'05-M'08) was born in Johannesburg, South Africa, on January 13, 1979. He received the B.Ing. (cum laude) and M.Ing. (cum laude) degrees in electric and electronic engineering from Rand Afrikaans University, Johannesburg, in 2001 and 2003, respectively, and the Ph.D. degree from Delft University of Technology, Delft, The Netherlands, in 2007.

Since 2007, he has been with KEMA Netherlands B.V., Arnhem, The Netherlands, as a Power Electronic Specialist. His current research interests include high-frequency dc-dc converters, integration technologies in printed circuit boards, and medium-voltage power converters for grid integration of renewable energy sources.

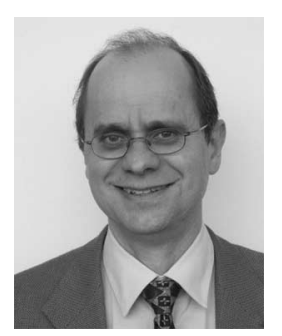

Braham J. A. Ferreira (F'05) was born in Pretoria, South Africa, in 1958. He received the B.Sc.Eng. (cum laude), M.Sc.Eng. (cum laude), and Ph.D. degrees in electrical engineering from Rand Afrikaans University, Johannesburg, South Africa, in 1980, 1982, and 1988, respectively.

During 1981, he was with the Institute of Power Electronics and Electric Drives, Technical University of Aachen, Aachen, Germany. From 1982 to 1985, he was with ESD (Pty) Ltd. From 1986 to 1997, he was with the Faculty of Engineering, Rand Afrikaans University, where he was the Carl and Emily Fuchs Chair of Power Electronics. Since 1998, he has been a Professor at the Electrical Engineering Mathematics and Computer Science Faculty, Delft University of Technology, Delft, The Netherlands.

Dr. Ferreira was the Chairman of the South African Section of the IEEE from 1993 to 1994. He is the Founding Chairman of the IEEE Joint Industry Applications Society (IAS)/Power Electronics Society (PELS) Benelux Chapter. He was the Transactions Review Chairman of the IEEE IAS Power Electronic Devices and Components Committee. He is an Associate Editor of the IEEE TRANSACTIONS ON POWER ELECTRONICS. He was a member of the IEEE PELS Adcom and is currently the Treasurer of the IEEE PELS. He was the Chairman of the Conference Internationale des Grands Reseaux Electriques (CIGRE) SC14 National Committee of the Netherlands and was a member of the Executive Committee of the European Power Electronics (EPE) Society.

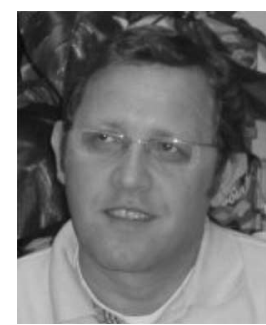

Pavol Bauer (SM'07) received the Master's degree in electrical engineering from the Technical University of Kosice, Kosice, Slovakia, in 1985, the Ph.D. degree from Delft University of Technology, Delft, The Netherlands, in 1995, and Prof. from Brno University of Technology, Brno, Czech Republic, in 2008.

Since 1990, he has been with Delft University of Technology, where he is engaged in teaching power electronics and electrical drives. From 2002 to 2003 , he was with KEMA, Arnhem, The Netherlands, where he was involved in different projects related to power electronics applications in power systems. He authored or coauthored over 40 journal and 170 conference papers, and five books. $\mathrm{He}$ also holds international patent, and has organized several tutorials at the international conferences. He has been involved in many projects for industry concerning wind power, power electronic applications for power systems such as Smarttrafo, etc., and has participated in several Leonardo da Vinci European Union (EU) projects as a project partner [Training in Electrical Engineering for Industry Automation (ELINA), Interactive and Unified E-Based Education and Training in Electrical Engineering (INETELE)] and a coordinator [E-Learning Distance Interactive Practical Education (EDIPE)].

Dr. Bauer is a Chapter Officer of the Benelux IEEE Joint Industry Applications Society and the Power Electronics and Power Engineering Society Chapter, and a member of the EPE and international steering committees of numerous conferences. 